\title{
Travel Location Recommendation from Users' Feedback
}

\author{
${ }^{1}$ Shree Laddha, ${ }^{2}$ Shailendra Aote \\ ${ }^{1,2}$ Shri Ramdeobaba College of Engineering and Management, Nagpur \\ Email: shreeladdha11@gmail.com
}

Received: 09 ${ }^{\text {th }}$ July 2018, Accepted: $14^{\text {th }}$ August 2018, Published: $31^{\text {st }}$ August 2018

\begin{abstract}
This paper discusses the method of travel sequence recommendation based on user's point of interests. Various methods and its limitations are discussed here, based on which, the proposed algorithm is provided. The entire process consist of following: Pages are available to the users based on page rank algorithm and users ${ }^{\text {ee }}$ feedback. Based on the point of interest, all the reviews are retrieved. The method is tested on self-created database consisting of user's feedback, user information, state and city, place and place types.
\end{abstract}

Keywords: Point of Interest, Recommendation, Page Rank Algorithm

\section{Introduction}

The travel recommendation is an important to suggest best location for user to travel as per its own point of interest. In this busy world our day to day life is getting more and more digital and social. All our daily need products can be ordered online and the products are delivered at your doorstep. In the same way travel sequence recommendation enables users to view the place of their interest and accordingly select the place to visit.

Our basic need is to get the best location from the user friend list and the best place to take in. The system that we design should recommend us the best restaurants, hotels, hospitality facility, accommodation and the best route to travel these places and the facilities in less time and in the minimum cost. It recommends the sequential path to travel that places in less time so that no wastage of time, money etc. These recommendations are recommended from the user friend list data that we save in our database which contains the description such as information, ratings, reviews, visiting time and season. Existing system and studies have not solved these problems. To collect all the correct and proper information of that place the user need to browse several websites and updates its original data. Several existing system might have different information about the same place so it become faulty. Recommendation from unknown resources may not have correct information so we proposing a system in which user point of interest is taken consideration through the user hobbies, preferences of the place type that user visit repeatedly. Such as place types are like trekking places, amusement park, water-park, forts, museums, rivers and dams, historical places, temples etc.
The existing study is not as advanced that can recommend the path to travel the places sequentially, and cannot recommend all the parameter in the same system. All these challenges we put it together and generate a well and suitable recommendation system which gives a sequential path for all the places that save time and cost of traveling. The paper is structured as follows; we introduce the literature review in section 2 . We describe how we do the implementation and proposed work which algorithms we use in our proposed system in section 3. Results of the implemented work are given in section 4. Conclusion is described in section 5.

\section{Literature Survey}

In this section, we define how we do related works

(1) Places recommendation from user's friend list from his profile and information in system, system like social media's such as Facebook, what's app, Instagram images etc.; (2) According the user's point of interest places recommendation; (3) Google map travel path route recommendation and place location recommendation. We also give the differences between our work and work done previously.

Graph based POI Recommendations:

In this paper the work done with breadth first preference propagation algorithm. The information for generating results is recommendation done with temporal influence and geographical temporal influence graph aware method and Also work done on path selection and time complexity analysis for generating appropriate recommendations. The dataset used is already generated which contains check-in records from Foursquare and Gowalla [1].

\section{Trajectories Based Recommendation:}

In this paper work is done in collaborative filtering which is used in mining trajectories. Amazon like POI trajectories are used for recommendation. The Longest Common Subsequence (LCS) approach is used to measure similarity based sequence relationship. The trajectories they used in this are from the Vienna zoo. The work done with three stages: (a) Building profile. (b) Computing Similarity. (c) Aggregating results and rating of similar user [2].

\section{Photo2Trip - Route recommendation from Geo-} Tagged photos:

In this paper work done with dynamic graph generation and dynamic programming for recommending the route the point cover for recommendation in this system are Destination discovering, internal path discovering, trip planning. The dataset of 20 million Photos is used from 
Panoramio. Path quality and similarity, fragment merging, nodes and edges discovering for graph generation, popular visiting time discovery, and destination style discovery are all the parameters are required for getting the final result [3].

Preference Aware Recommendation From Sparse Social Networking Data:

In this paper work is done with preferences of user location from user information. Collect preferences from user inferring rating. The dataset used has a structure with user data, check-in, check-out, venue (place). System work in both the sense offline and online, where offline system considers two parts,

(a) Social knowledge learning, (b) Preference discovery, where online system considers two parts

(a) Location selection, (b) Preference aware candidate selection [4].

\section{Personalized Travel Sequence Recommendation} Algorithm

First we implement page rank algorithm which we use to find the effective ranking for the location search by user, which can be calculated from ratings given by other user that can be done in process as follows:

Page Rank: Page Rank is used for Ranking all the complete graph and implement search. Ranks pages based on the number of backlinks pointing to them.

\section{Working of Page Rank}

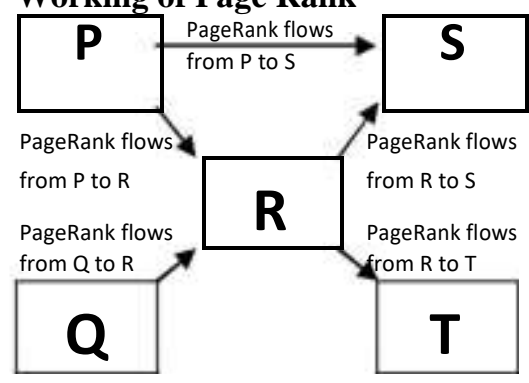

Fig 1 Flow of Page Rank Algorithm

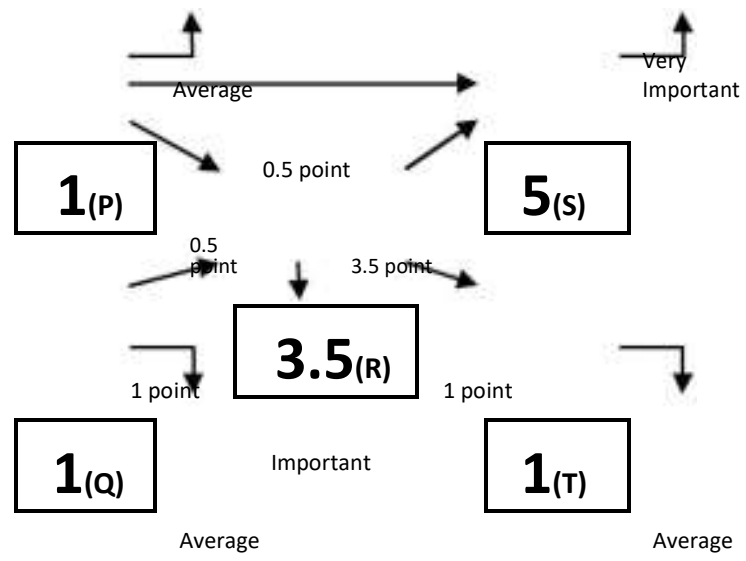

Fig 2a Example of Page Rank

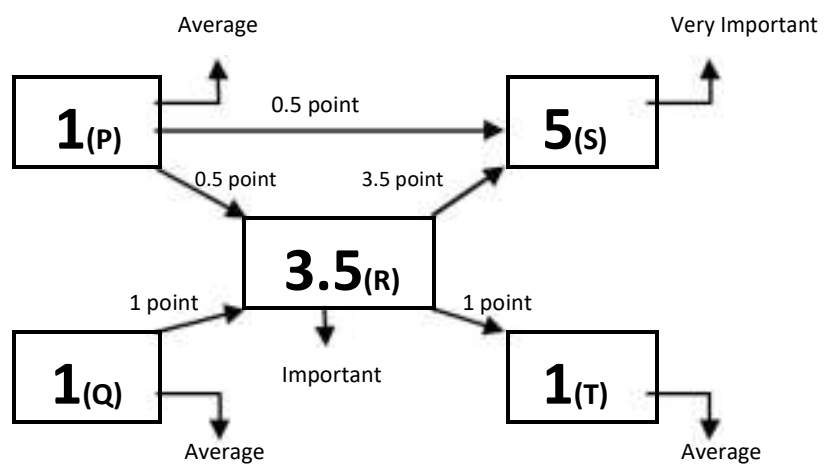

Fig 2b Example of Page Rank Algorithm

- $\quad$ Page $\mathrm{S}$ will be on the Top Result because it has a Page Rank of 5

- $\quad$ Page R will be on the $2^{\text {nd }}$ Result because it has a Page Rank of 3.5

- $\quad$ Page P, Q \& T will be on the Top Result because they have a Page Rank of 1 (as shown in Fig. 2)

Page Rank algorithm is an algorithm uses to measure the importance of website pages using hyperlinks between pages.

Some hyperlinks point to pages to the same site (in links) and other point to pages in other websites (out links) (as shown in Fig. 1)

Page Rank is a "vote", by all the other pages on the web, about how important a page is important.

A link to a page counts as a vote of support.

The Main Concepts:

In links of page $\mathrm{i}$ : These are the hyperlinks that points to page $\mathrm{i}$ from other pages. Usually, hyperlinks from the same site are not considered

Out links of page i: These are the hyperlinks that point out to other pages from page $\mathrm{i}$.

$$
\boldsymbol{P}(\boldsymbol{i})=\sum_{(j, i) \varepsilon E} \frac{P(j)}{O j}
$$

$\mathrm{P}(\mathrm{i})=$ Page Rank score of page $\mathrm{i}$

$\mathrm{O} \mathrm{j}=$ Number of out links of page $\mathrm{j}$

Hyperlink Induced Topic Search:

HITS algorithm uses a simple approach to finding quality documents and assumes that if document $\mathrm{A}$ has a hyperlink to document $\mathrm{B}$, then the author of document $\mathrm{A}$ thinks that document $\mathrm{B}$ contains valuable information. In $A$ is seen to point to a lot of good documents, then $A^{\text {ee }} \mathrm{S}$ opinion become more valuable and the fact that $\mathrm{A}$ point to B would suggest that B is a good document as well.

Second we implement HITS algorithm which we use to find the effective review for the location search by user, which can be calculated from reviews given by other user that can be done in process as follows: 


\section{Steps of HITS Algorithm:}

Starting from a user supplied query, HITS assembles an initial set $S$ of pages.(as shown in Fig. 3). The initial set of pages is called root set.

These pages are then expanded to a larger root set $\mathrm{T}$ by adding any pages that are linked "to or from" any page in the initial set S. (as shown in Fig. 4)

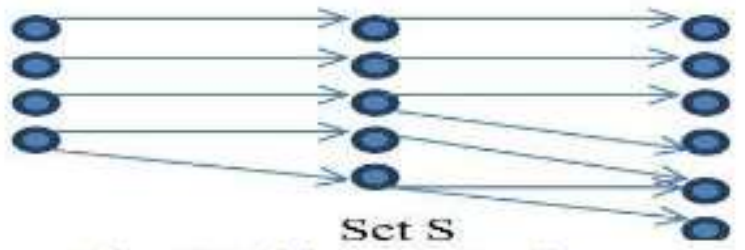

Fig. 3 Initial set $S$ of pages

HITS then associates with each page $\mathrm{P}$ a hub weight $\mathrm{h}(\mathrm{p})$ and an authority weight $\mathrm{a}(\mathrm{p})$, all initialized to one.

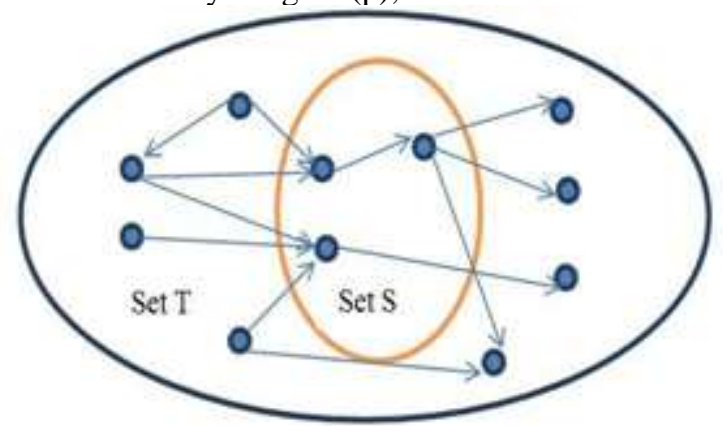

Fig. 4 Set T expanded by adding pages linked "to and from" the pages in the initial set S

HITS then iteratively updates the hub and authority weights of each page.

Let $\mathrm{p} \square \mathrm{q}$ denotes "page $\mathrm{p}$ has an hyperlink to page q" HITS update the hubs and authorities as follows:

$a(p)=\sum_{p \rightarrow q} h(q)$

$h(p)=\sum_{q \rightarrow p} a(q)$

\section{HITS Algorithm Applies Two Main Steps:}

A sampling component which constructs a focused collection of thousands web pages likely to be rich in authorities.

A weights propagation component which determines the numerical estimates of hub and authority weights by an iterative procedure.

\section{Results and Discussion}

We have created our own data set by analysing the interest of the users. This data set mainly focuses on user's point of interest. The description of our selfcreated data set is given below:
First we have created a data set of the registered user as shown in fig. 5. When the user registers itself with the system then only he will get full access to the system in which he can updated his profile, create his own friend list, share his experience and he can give his feedback for the places he visited. User needs to give its email address and mobile number for verification which also determines the uniqueness of the user. No duplication is allowed.

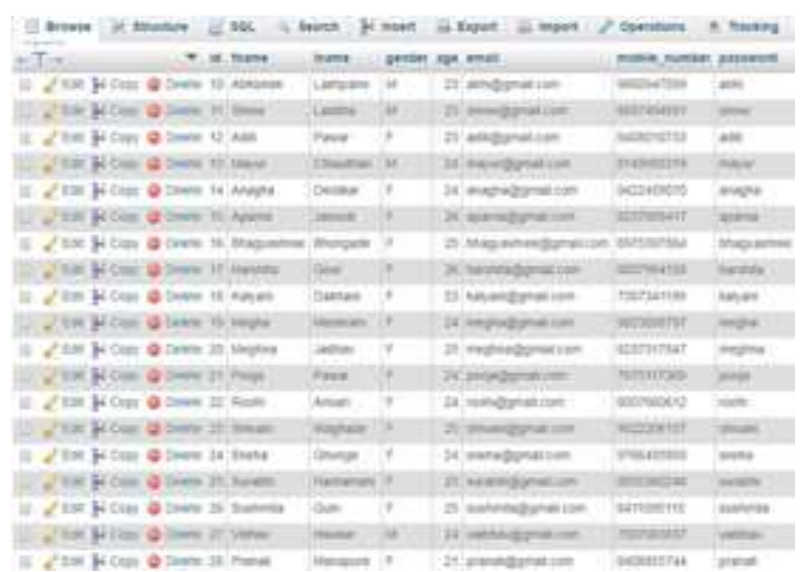

Fig 5 Data Set of the Registered Users

Second we have created a data set for places that can be visited in a particular location. The data set for some places for Nagpur city location are shown in the fig. 6, for which the GUI of these places is represented in fig. 7. Here the user needs to register itself to the system and give his point of interest by selecting country, state and city which he wants to visit. Then the list of places along with their ratings is shown.

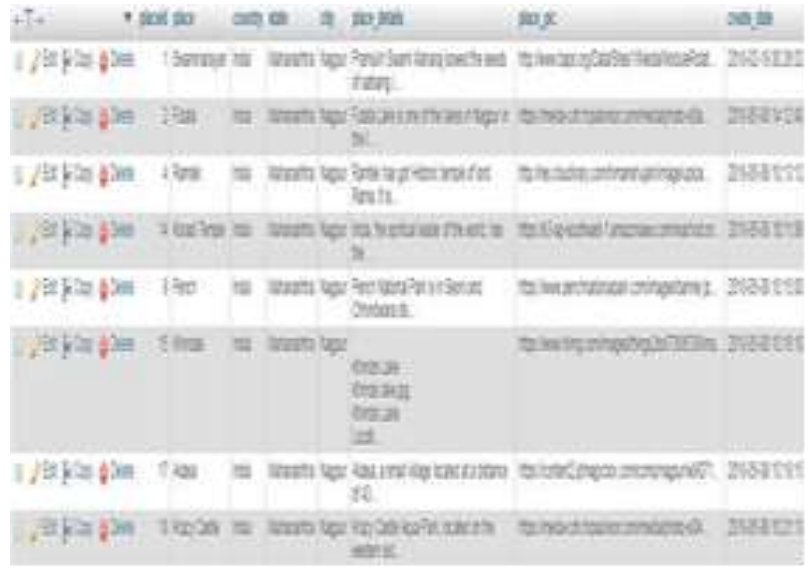

Fig 6 Data Set of the Places in Nagpur 


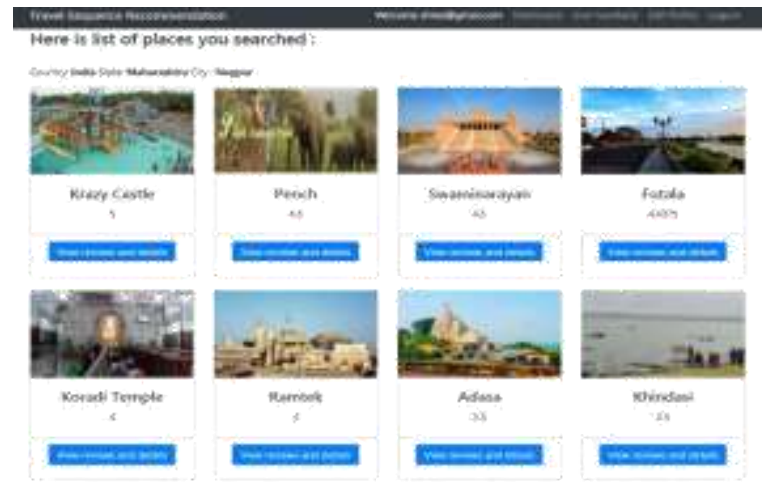

Fig 7 GUI of the Places in Nagpur with Their Ratings

When we select a particular place from the list of places then the full description of that place, its sample image and reviews of the previously visited user of that place is given.(As shown in fig. 8)

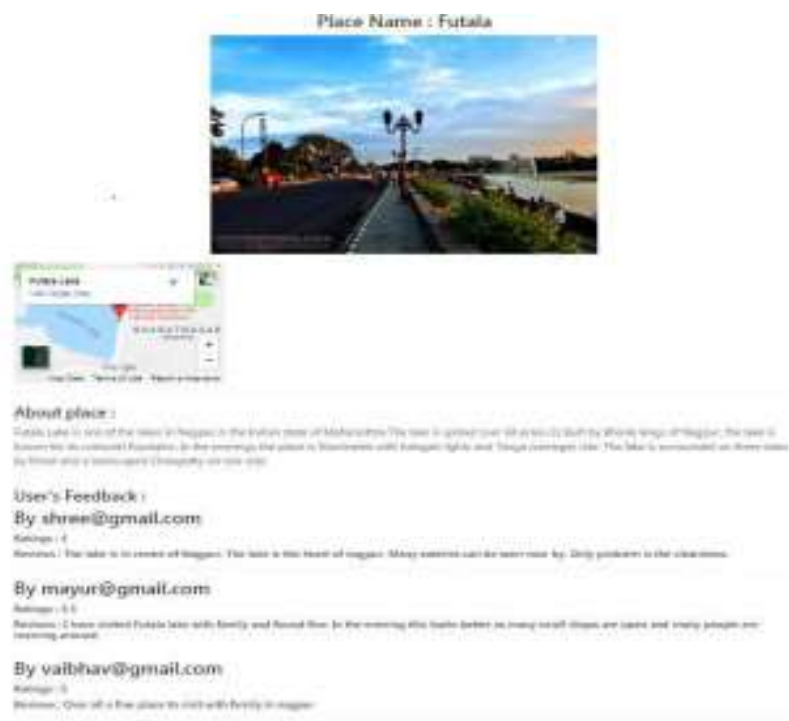

Fig 8 Details of the Selected Place

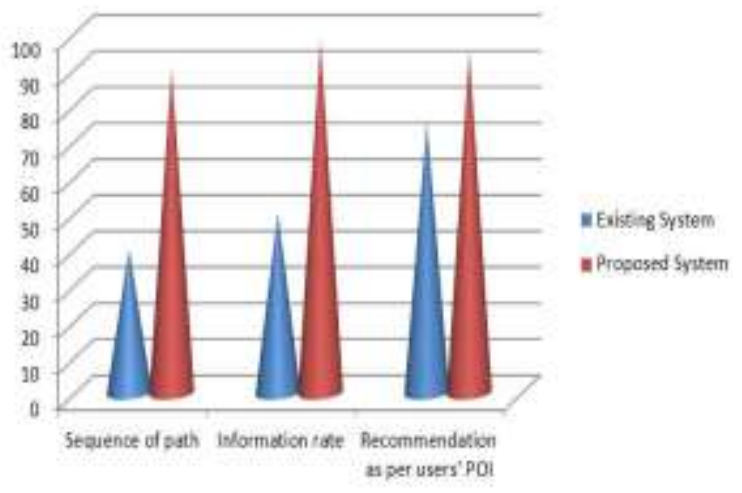

Fig.9 Accuracy of our Recommendation System
The diagram above shows the comparison between the existing system and the proposed system. From which we come to know that we have boost up the accuracy rate in our work. The system is power enough that the information given by it is accurate

\section{Conclusion}

In this paper, we propose Travel Sequence Recommendation from User's Point of Interest, which is powerful enough to recommend the best location to travel using collaborative filtering technique based on user's point of interest. It provides most accurate information and instant answers to user about the location he searches. The system is finally able to suggest the most suitable location for traveling according to user's interest in less time. The system gives best time, best season to visit the place and the shortest route to reach that place.

\section{References}

[1] Q. Yuan, G. Cong, and A. Sun, "Graph-based point-of-interest recommendation with geographical and temporal influences," in Proc. 23rd ACM Int. Conf. Inform. Knowl. Manage. , 2014, pp. 659-668.

[2] Haosheng Huang and Georg Gartner Institute of Geoinformation and Cartography, Vienna University of Technology, Karlsplatz 13, 1040 Vienna, Austria, "Using trajectories for collaborative filtering-based POI recommendation" Int. J. Data Mining, Modelling and Management, Vol. 6, No. 4, 2014.

[3] X. Lu, C. Wang, J. Yang, Y. Pang, and L. Zhang, "Photo2trip: Generating travel routes from geo-tagged photos for trip planning," in Proc. Int. Conf. Multimedia, 2010, pp. 143152.

[4] Jie Bao, Yu Zheng, David Wilkie, and Mohamed Mokbel "Recommendations in Location-based Social Networks:A Survey, Geoinformatica, 6 February 2015.

[5] Desrosiers, C., and Karypis, G. (2010) „A comprehensive survey of neighborhood-based recommendation methods ${ }^{\text {ee }}$, in Ricci, F. et al (Eds.), Recommender Systems Handbook, Springer, pp. 107-144.

[6] Akshitha Sivakumar and B Prabadevi, "Tour Recommendation Guide- Personalized travel sequence recommendation" in IOP Conference Series: Material Science and Engineering, vol. 263, Computation and Information Technology, 2017.

[7] Letchner, J., Krumm, J. and Horvitz, E. (2006),,Trip Router with Individualized Preferences (TRIP): Incorporating 
personalization into route planningee in Proceedings of the IAAI ${ }^{\mathrm{ee}} 06$, AAAI Press, pp. 1795-1800.

[8] Zheng, Y., Zhang, L., Xie, X., and Ma. W. (2009a) ,Mining interesting locations and travel sequences from GPS trajectories ${ }^{\text {ce }}$ in Proceedings of WWW 2009, ACM Press, pp. 791-800.

[9] H. Kori, et al. Automatic Generation of Multimedia Tour Guide from Local Blogs. In MMM, 2007.

[10] Q. Hao and R. Cai, et al. Generating Location Overviews with Images and Tags by Mining User-Generated Travelogues. In ACM MM, 2009.

[11]Zahra Farzanyar and Nick Cercone, "Trip Pattern Mining Using Large Scale Geo-tagged Photos" in International Conference on Computer and Information Science and Technology, Paper no. 113, May 12, 2015.

[12] T. Kurashima, T. Tezuka, and K. Tanaka, "Mining and visualizing local experiences from blog entries," in Database and Expert Systems Applications. New York, NY, USA: Springer, 2006, pp. 213-222.

[13] H. Huang and G. Gartner, "Using trajectorie for collaborative filtering-based POI recommendation," Int. J. Data Mining, Modelling Manage., vol. 6, no. 4, pp. 333-346, 2014.

[14]P. Lou, G. Zhao, X. Qian, H. Wang, and X. Hou, "Schedule a rich sentimental travel via sentimental POI mining and recommendation," in Proc. 20th ACM Int. Conf. Multimedia Big Data, 2016, pp. 33-40.

[15]Zheng Y., Liu L., Wang L. and Xie X. Learning transportation modes from raw GPS data for geographic applications on the Web. In Proc. of the 17th Intl. Conf. on World Wide Web (Beijing, China, 2008), ACM Press: 247-256.

[16] Jie Bao, Yu Zheng, David Wilkie, and Mohamed Mokbel "Recommendations in Location-based Social Networks:A Survey, Geoinformatica, 6 February 2015.

[17] J. Li, X. Qian, Y. Y. Tang, L. Yang, and T. Mei, "GPS estimation for places of interest from social users"e uploaded photos," IEEE Trans. Multimedia, vol. 15, no. 8, pp. 2058-2071, Dec. 2013.

[18] S. Jiang, X. Qian, J. Shen, Y. Fu, and T. Mei, "Author topic model based collaborative filtering for personalized POI recommendation," IEEE Trans. Multimedia, vol. 17, no. 6, pp. 907-918, Jun. 2015.

[19]Jiang Shuhui, Qian Xueming, Shen Jialie, Fu Yun and Mei Tao 2015, "Model- Based
Collaborative Filtering for Personalized POI Recommendations" IEEE Trans. Multimedia 17 907-918.

[20] Li Jing, Qian Xueming, Tang Yuan Yan, Yang Linjun and Mei Tao 2013, “GPS Estimation for Places of Interest From Social Users' Uploaded Photos" IEEE Trans. Multimedia 15 20582071.

[21]Farzanyar Z, and Cercone N. (2013)"Accelerating Frequent Itemsets Mining on the Cloud: A MapReduce-Based Approach." Data Mining Workshops (ICDMW), 2013 IEEE 13th International Conference on. IEEE. 